\title{
Safety and Efficacy of Enoxaparin in Pregnancy: A Systematic Review and Meta-Analysis
}

\author{
Barry Jacobson - Virendra Rambiritch • Dara Paek - Tobias Sayre • \\ Poobalan Naidoo · Jenny Shan · Rory Leisegang
}

Received: September 23, 2019 / Published online: October 31, 2019

(c) The Author(s) 2019

\begin{abstract}
Introduction: International guidelines support the use of low molecular weight heparins for the treatment of thromboembolism and thromboprophylaxis during pregnancy. However, evidence of the benefit and harm associated with specific low molecular weight heparins such as
\end{abstract}

Enhanced Digital Features To view enhanced digital features for this article go to https://doi.org/10.6084/ m9.figshare.9933413.

Electronic supplementary material The online version of this article (https://doi.org/10.1007/s12325019-01124-z) contains supplementary material, which is available to authorized users.

\section{B. Jacobson}

Department of Haematology and Molecular Medicine, University of Witwatersrand, 1 Jan Smuts Ave, Johannesburg, South Africa

V. Rambiritch

Department of Pharmacology, University of

KwaZulu-Natal, University Rd, Westville, South

Africa

D. Paek · T. Sayre

Doctor Evidence, 301 Arizona Ave, Santa Monica, CA, USA

P. Naidoo

Sanofi Affiliate, Medical, 2 Bond St, Grand Central, Midrand, South Africa enoxaparin is dated. No current systematic review and meta-analysis describing the safety and efficacy of enoxaparin for thromboembolism and thromboprophylaxis during pregnancy exists.

Methods: PubMed, Embase, and Cochrane databases were searched on August 17, 2018 for clinical trials or observational studies in pregnant women receiving enoxaparin; patients with a prosthetic heart valve were excluded. Risk ratios (RR) with 95\% confidence intervals (CI) were calculated using a random effects model, and heterogeneity was measured using the $I^{2}$ statistic.

P. Naidoo

Department of Health Informatics, School of Health Professions, Rutgers, State University of New Jersey, 57 US Highway 1, New Brunswick, NJ, USA

J. Shan

Sanofi Global, Medical, 54-56 Rue la Boétie, Paris, France

R. Leisegang $(\varangle)$

Department of Pharmaceutical Biosciences, Uppsala University, 75236 Uppsala, Sweden e-mail: rory.leisegang@gmail.com

R. Leisegang

FAMCRU, The Department of Paediatrics \& Child Health, Stellenbosch University, Tygerberg, Cape Town, South Africa 
Results: Of the 485 records identified in the search, 24 studies published clinical trials, and observational studies were found dating back to 2000. Only one observational cohort and one randomized control trial focused on the use of enoxaparin for thromboprophylaxis and therefore efficacy was not assessed; the other studies included women with recurrent pregnancy loss (15 studies), history of placental vascular complications (five studies), and recurrent in vitro fertilization failure (two studies) and were therefore analyzed in terms of safety only. Bleeding events were non-significantly more often reported for enoxaparin compared to untreated controls (RR 1.35 [0.88-2.07]) but less often reported for enoxaparin versus aspirin (RR 0.93 [0.62-1.39]); thromboembolic events, thrombocytopenia, and teratogenicity were rarely reported events; in patients with a history of recurrent pregnancy loss, encouragingly the rates of pregnancy loss were significantly lower for enoxaparin compared to untreated controls (RR 0.58 [0.34-0.96]) and enoxaparin + aspirin versus aspirin alone (RR 0.42 [0.32-0.56]) as well as observably lower for enoxaparin versus aspirin alone (RR 0.39 [0.15-1.01]), though significant heterogeneity was observed $\left(I^{2}>60\right)$.

Conclusion: Literature on the efficacy and safety of enoxaparin for thromboembolism and thromboprophylaxis remains scanty, and therefore efficacy was not assessed; in terms of safety, when including other indications for enoxaparin in pregnancy, we found that enoxaparin was associated with significantly lower complications than aspirin. Given differences in study design and study heterogeneity, pregnancy loss results should be interpreted with caution. Moreover, reports of thromboembolic events, thrombocytopenia, and congenital malformations were rare.

Funding: Sanofi.

Keywords: Cardiology; Enoxaparin; Low molecular weight heparin (LMWH); Pregnancy; Thromboembolism; Thromboprophylaxis; Venous thromboembolic events (VTE)

\section{Key Summary Points}

Venous thromboembolic (VTE) disease is a major cause of morbidity and mortality during pregnancy and the puerperium. Enoxaparin, a low molecular weight heparin (LMWH), is standard of care for the management of VTE risk in pregnancy, after individualized benefit-risk assessment. This systematic review and meta-analysis demonstrate the safety of enoxaparin in pregnancy.

Though reports of thromboembolic events, thrombocytopenia, and teratogenicity were rare, enoxaparin use during pregnancy was associated with lower occurrence of pregnancy loss when compared with controls and similar rates of bleeding.

Both real-world studies and clinical trials were assessed in this analysis, and, in light of the adoption of antenatal LMWH in clinical practice, real-world data and continued safety reporting will help further inform future treatment guidelines surrounding this important topic.

\section{INTRODUCTION}

Pregnancy is a pro-thrombotic state and has been associated with a fourfold increase in the risk of thromboembolic events [1, 2]. Venous thromboembolic events (VTE), which include deep vein thrombosis (DVT) and pulmonary embolism (PE), are one of the leading causes of maternal mortality in developed countries $[3,4]$. Treatment options for prevention and treatment of VTE are limited, because of the maternal and fetal risk of anticoagulation, and only a limited number of drugs are considered safe and prescribed during pregnancy.

Unfractionated heparin (UFH) and low molecular weight heparin (LMWH) do not cross the placenta and pose low risk of fetal exposure 
$[5,6]$. LMWHs have potential benefits over UFH, such as higher bioavailability, longer halflife, and more predictable response. These factors together allow for once or twice daily administration with minimal laboratory monitoring, which makes LMWH ideal for outpatient use [5]. International guidelines now support the use of LMWH over UFH and other oral anticoagulant agents for the treatment of VTE and thromboprophylaxis in pregnancy following appropriate risk factor assessment [7-13]. However, recommendations are largely based on consensus or expert opinion, and further high-quality research is still needed to guide clinical decisions.

Systematic reviews and meta-analyses which have examined antenatal LMWH for VTE in terms of the benefit and harm associated with specific LMWHs such as enoxaparin are dated [14]. The aim of this review was to update the evidence surrounding the safety and efficacy of enoxaparin in pregnancy, focusing on the thromboprophylaxis efficacy and safety, but including other indicators in terms of safety.

\section{METHODS}

A systematic literature review was conducted following a standardized review protocol using the PICOTSS framework, which outlined the population, interventions, comparators, outcomes, timing, setting, and study designs of interest. This review followed standard methodology for conducting systematic reviews as per guidelines provided by the Centre for Reviews and Dissemination [15], National Institute for Health and Care Excellence [16], and the Cochrane Handbook for Systematic Reviews of Interventions [17]. Results were reported according to the Preferred Reporting Items for Systematic Reviews and Meta-Analyses (PRISMA) guidelines [18].

This review is based on previously conducted studies and does not contain any studies with human participants or animals performed by any of the authors.

\section{Literature Search}

A comprehensive search was conducted in PubMed, Embase, and the Cochrane Register of Controlled Trials for publications available up to August 17, 2018. Conference proceedings from 2015 to 2018 were also searched through Embase. The search strategies are provided in Table S1 of the Electronic Supplementary Material. Electronic literature searches were performed by medical librarians from Doctor Evidence, LLC using a proprietary software platform (Doctor Evidence: Library Management System. Santa Monica, CA: Doctor Evidence, LLC) [19].

\section{Study Selection}

Given the particular concerns around maternal and fetal safety, we explored whether any antenatal use of enoxaparin has been associated with increased risks of adverse outcomes. Studies were included in the review if they met the following criteria: (1) study population of pregnant women; (2) clinical trials or observational studies examining antenatal use of enoxaparin compared to another non-enoxaparin group including placebo; (3) outcomes included incidence of thromboembolic events, bleeding, thrombocytopenia, pregnancy loss, and congenital malformations. To summarize the available literature on the potential benefits and harms of enoxaparin use during pregnancy, women requiring thromboprophylaxis or other pregnancy-related indications were included. Patients with mechanical heart valves were excluded. The study eligibility criteria are provided in Table S2 (Electronic Supplementary Material).

The initial screening of references retrieved in the search was performed by two independent medical librarians to identify potentially relevant studies based on the titles and abstracts. The full texts of studies were then reviewed, and detailed reasons for inclusion or exclusion were documented. The initial agreement was above $85 \%$. Any disagreements were resolved by discussion or an independent thirdparty reviewer. 


\section{Data Extraction and Quality Assessment}

Data extraction included study-level characteristics, treatment information, and the prespecified safety/efficacy outcomes of interest. Data extraction was carried out by two clinical data associates using the Digital Outcome Conversion (DOC) Data Version 2.0 Software platform (Doctor Evidence, LLC, Santa Monica, CA, USA). All characteristic and outcome terms were collected as reported in each paper, and synonyms were bound using the DOC Ontology System (DOC Data version 2.0. Santa Monica, CA: Doctor Evidence, LLC).

Quality assessments were conducted by two independent reviewers, and any disagreement between the reviewers was resolved by discussion. Data were stored and managed in Microsoft Excel. The Cochrane Collaboration tool for assessing the risk of bias in randomized trials was used to assess clinical trials [20]. This instrument has been used to evaluate seven domains of bias: random sequence generation (selection bias), allocation concealment (selection bias), blinding of participants and personnel (performance bias), blinding of outcome assessment (detection bias), incomplete outcome data (attrition bias), selective reporting (reporting bias), and other sources of bias (other bias). The quality of observational cohort studies was assessed using the Newcastle-Ottawa Scale (NOS) [21]. The NOS assigns up to a maximum of 9 points (or stars) for the least risk of bias in three categories: (1) Selection of study groups (maximum 4 points); (2) Comparability of study groups (maximum 2 points); and (3) Outcome ascertainment (maximum 3 points). For the second item in the outcome category, "2. Was follow-up long enough for outcomes to occur?", a follow-up of 40 weeks (9 months) or more was considered adequate. For the third item in the outcome category, "3. Adequacy of follow-up of cohorts", a follow-up rate of at least $90 \%$ or a description provided of those lost was considered adequate to assign a point.

\section{Analysis}

Analyses were conducted for comparisons of enoxaparin (alone and in combination with aspirin) versus placebo/untreated controls, aspirin, and other anticoagulation comparator groups where there was sufficient data reported. Traditional pairwise meta-analyses were performed for the outcomes of bleeding and pregnancy loss. Only qualitative analyses were feasible for thromboembolic events, thrombocytopenia, and congenital malformations. Relative risk/risk ratios and $95 \%$ confidence intervals for outcomes were determined using a random effects model. For studies that reported no events for a single treatment arm, a conservative statistical continuity correction was applied by assigning the group a 0.5 event value. Studies that reported no events in both treatment arms were excluded from the metaanalysis. Heterogeneity between studies was estimated using the $I^{2}$ statistic. If significant heterogeneity was observed $\left(I^{2}>50\right)$, differences in study design, single vs. multicenter setting, and primary indication were examined.

\section{RESULTS}

A total of 485 records were identified in the search, of which 422 records were excluded during title/abstract screening. On the basis of a review of the full texts of 63 studies, an additional 39 studies were excluded, primarily for inappropriate comparison (i.e., no comparison between different treatments) and for not reporting the outcomes of interest. Twenty-four studies met eligibility criteria and were included for data extraction. The PRISMA flow diagram is presented in Fig. 1.

There were 18 randomized controlled trials (RCTs), two prospective non-randomized studies, two prospective observational studies, and two retrospective observational studies, with publication dates ranging between 2000 and 2017. Study populations included women with recurrent pregnancy loss (15 studies), history of placental vascular complications (five studies), recurrent in vitro fertilization failure (two studies), and thromboprophylaxis (two studies). The characteristics of studies and reported outcomes are summarized in the Electronic Supplementary Material (Table S5). 
Twenty publications [22-41], pertaining to 19 clinical trials [22-33, 35-41] were included in the Cochrane risk of bias assessment. A summary of the assessment for the randomized trials is presented (Table S3 in the Electronic Supplementary Material). The risk of bias across the included studies was generally low and limited to the blinding of participants and personnel (performance bias) and the blinding of outcome assessment (detection bias): 14 studies were judged to be at low risk for the random sequence generation item in the selection bias domain [23-33, 38-40], three studies were judged as unclear, as the random sequence generation methods were not clearly described $[22,35,40]$, and four studies were considered high risk as they were non-randomized $[36,37,42,43]$. Regarding allocation concealment, 14 studies were considered to be at low risk of bias [22-25, 27, 28, 30-32, 35, 36, 38, 40, 41], four studies were considered unclear as methods for allocation concealment were not provided or not clearly described, one study was considered as high risk as randomization was not carried out and the groups were made aware of the treatment they received upon enrollment [37]. Few studies presented adequate blinding methods to reduce performance bias: only three studies were considered as low risk for sufficiently packaging their treatments to disguise their identities to patients receiving them at all points in the study $[28,38,41]$, eight studies were considered unclear as blinding methods were not provided, ten studies were open-label for participants and personnel and were thus considered to be at high risk for performance bias [22, 24, 25, 27, 30-33, 37, 40]. Similarly, for detection bias, only five studies were considered low risk [28, 30, 38, 39, 41], six studies were of unclear risk as the study was not described as either open-label or blinded $[23,29,35,36,42,43]$, and nine studies were considered high risk because of an open-label study design [22, 24-27, 31, 33, 37, 40]. All studies were considered as low risk in the domains of incomplete outcome data (attrition bias) and selective reporting (reporting bias). Most studies used an intent-to-treat analysis in their primary outcome analysis, and where dropouts were present, the numbers were few and similar between groups. All studies reported on their prespecified primary outcome, as mentioned in the publication. Finally, no other sources of bias other than the ones previously described were detected across the studies.

Four studies were included in the NOS quality assessment, all of which were cohort studies [42-45]. A summary of the assessment is presented in the Electronic Supplementary Material (Table S4). One study had a score of 8 and three studies scored the maximum score of 9. All studies scored the maximum score of 4 stars in the selection category, indicating that the data used was representative of the targeted community. For the comparability category, one study scored only one star because the authors did not describe any additional baseline characteristics that were well matched between cohorts [42] and the other three studies scored the maximum score of 2 stars [43-45]. Finally, in the outcome category, all studies scored the maximum of 3 stars.

\section{Thromboembolic Events: Efficacy and Safety}

Only two studies looked at enoxaparin in the context of thromboprophylaxis; a pooled metaanalysis was therefore not possible.

In terms of safety of enoxaparin, a pooled meta-analysis for thromboembolic events was not feasible because of the low number of reported cases across treatment comparisons. Data reported in the studies are summarized in Table S6 (Electronic Supplementary Material). Two cases of DVT were reported in patients with recurrent pregnancy loss who received enoxaparin monotherapy compared to four cases in patients who received no intervention (RR 0.5; 95\% CI 0.09-2.69) [22]. No thromboembolic events were reported in patients receiving combined therapy with enoxaparin plus aspirin. In one study that compared enoxaparin plus aspirin versus aspirin in women with a history of preeclampsia, one case of superficial venous thrombosis was reported in patients who received aspirin [34]. 


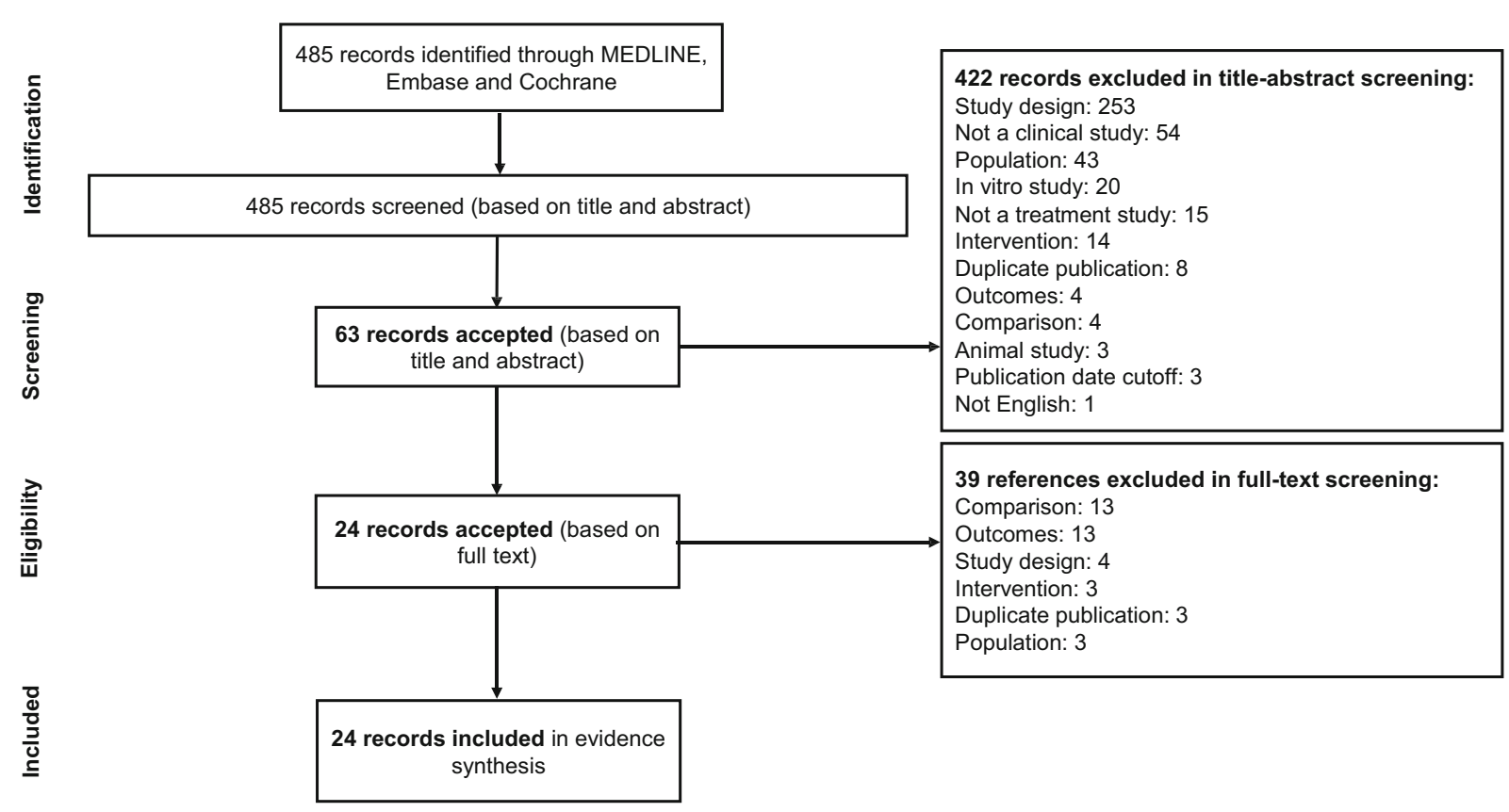

Fig. 1 PRISMA diagram

\section{Bleeding}

Results of the pooled meta-analyses for bleeding are presented in Table 1 and Fig. 2. The incidence of bleeding events with enoxaparin monotherapy was 35\% higher compared to placebo/no treatment controls. When compared to aspirin, risk of bleeding was 7\% lower with enoxaparin monotherapy and 5\% lower with enoxaparin plus aspirin. These results were not statistically significant, and heterogeneity between the studies was low. Enoxaparin was compared to tinzaparin in one study in women with recurrent pregnancy loss [45]. No bleeding events were reported in either treatment group. In another study in women with recurrent pregnancy loss who also tested positive for antiphospholipid antibodies, three bleeding events were reported in patients receiving enoxaparin plus aspirin compared to two events in those who received UFH plus aspirin [37]. Bleeding event data are summarized in Table S7 (Electronic Supplementary Material).

\section{Pregnancy Loss}

Results of the pooled meta-analyses for pregnancy loss are presented in Table 1 and Fig. 3.
Risk of pregnancy loss was reduced by $58 \%$ when enoxaparin monotherapy was compared to control groups, and significant heterogeneity was observed (Fig. 3a). Though there was a large numerical difference, reduction in pregnancy loss for enoxaparin monotherapy compared to aspirin did not achieve statistical significance (Fig. 3b). A sensitivity analysis removing one study with no events in the enoxaparin group did not change the result. Pregnancy loss was significantly reduced by $42 \%$ with enoxaparin plus aspirin compared to aspirin alone $(p<0.0001)$ (Fig. 3c). In a sensitivity analysis based on the study design, a lower risk ratio was found for pregnancy loss in the observational data than the RCT data. Enoxaparin plus aspirin was still statistically favored over aspirin and the study design did not appear to influence this result. A non-statistically significant reduction of $66 \%$ was found when enoxaparin plus aspirin was compared to UFH plus aspirin (Table 1). Pregnancy loss reported in the studies is summarized in Table S8 (Electronic Supplementary Material). 


\section{Thrombocytopenia}

Few cases of thrombocytopenia occurred in the studies (Table S9 in the Electronic Supplementary Material). In two RCTs in women with recurrent pregnancy loss, ten events were reported for enoxaparin and three events for placebo [22, 38]. Thrombocytopenia with enoxaparin plus aspirin was reported in one study, which reported two cases for enoxaparin plus aspirin and four cases for aspirin, in women with a history of preeclampsia [34].

\section{Congenital Malformations}

Eight cases of congenital malformations were reported for enoxaparin, two cases for aspirin, and five cases for placebo in three RCTs in women with recurrent pregnancy loss $[24,26,38]$. In an observational study in

Table 1 Pooled relative risks of bleeding events and pregnancy loss

\begin{tabular}{llc}
\hline Outcome & $\begin{array}{c}\text { No. of } \\
\text { studies }\end{array}$ & $\begin{array}{c}\text { RR }(95 \% \text { CI }) \\
\text { Random effects; } \\
\boldsymbol{I}^{\mathbf{2}}\end{array}$ \\
\hline Bleeding & & $1.35(0.88-2.07) ;$ \\
$\begin{array}{l}\text { Enoxaparin vs } \\
\text { placebo/controls }\end{array}$ & 5 & $I^{2}=16.05$ \\
Enoxaparin vs aspirin & 3 & $0.93(0.62-1.39) ;$ \\
& & $I^{2}=0$ \\
Enoxaparin + aspirin vs & 4 & $0.95(0.71-1.28) ;$ \\
aspirin & & $I^{2}=11.28$ \\
Pregnancy loss & & $0.58(0.34-0.96) ;$ \\
Enoxaparin vs & 6 & $I^{2}=65.80$ \\
placebo/controls & & $0.39(0.15-1.01) ;$ \\
Enoxaparin vs aspirin & 4 & $I^{2}=78.24$ \\
& & $0.42(0.32-0.56) ;$ \\
Enoxaparin + aspirin vs & 8 & $I^{2}=14.10$ \\
aspirin & & $0.66(0.33-1.34) ;$ \\
Enoxaparin + aspirin vs & 2 & $I^{2}=0$ \\
UFH + aspirin & &
\end{tabular}

women with a history of obstetric and/or thromboembolic complications, single cases of congenital malformations were reported in patients receiving enoxaparin plus aspirin in the first trimester or aspirin only. There were no cases observed with second trimester enoxaparin plus aspirin (Table S10 in the Electronic Supplementary Material).

\section{Maternal Mortality}

Maternal mortality was reported in one study and no deaths were reported in either of enoxaparin plus aspirin and aspirin groups during the study.

\section{Allergic Reaction}

Allergy or skin reactions were reported in three studies. One of the studies reported no allergy, and the remaining two studies reported around one to four more cases of allergic reaction when compared to the standard care or aspirin groups.

\section{Study Withdrawal}

Study withdrawal was reported in eight studies. However, five reported zero discontinuations in the remaining three studies, and each of them reported a unique treatment comparison involving enoxaparin.

\section{DISCUSSION}

The findings of this systematic review and metaanalysis were that antenatal enoxaparin use was generally safe, but that enoxaparin efficacy specifically for antenatal VTE could not be examined as a result of only two studies meeting the inclusion criteria. Given that the use of enoxaparin in pregnancy remains off-label, careful individual benefit-risk assessment and informed consent are still required before its use. In terms of efficacy. These findings are aligned with those of the review conducted by Greer et al. [14] in LMWH in general. 
a Study

Risk Ratio (95\% CI)

\begin{tabular}{lll}
\hline Badawy AM (2008) \\
Fawzy M (2008) \\
Gris JC (2010) \\
Pasquier E (2015) \\
Oublan H (2008) \\
RE Model \\
Favors Enoxaparin
\end{tabular}

b Study

Risk Ratio (95\% CI)

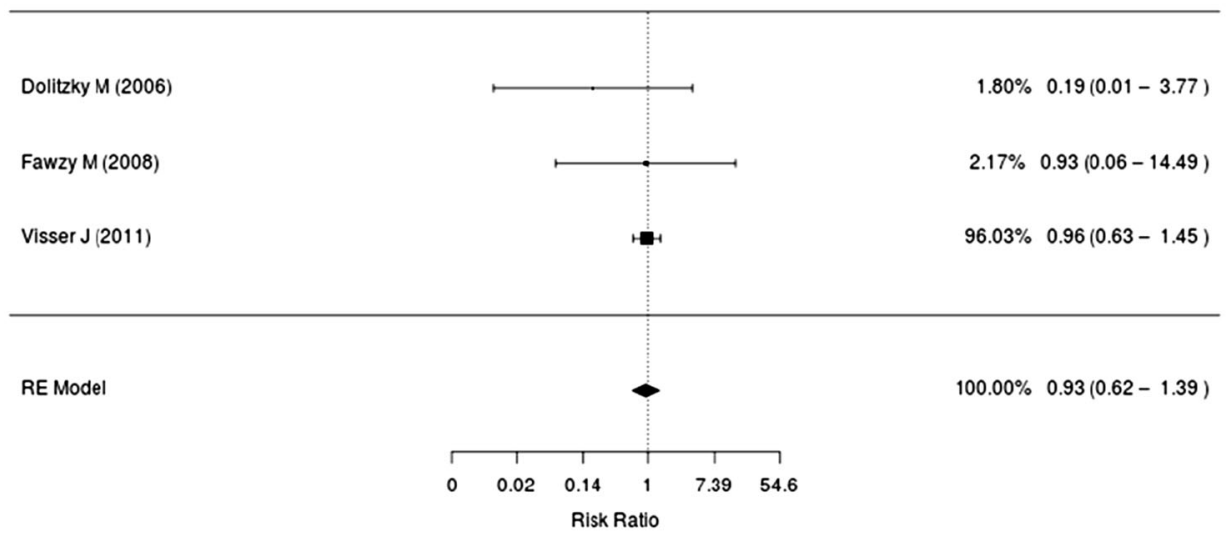

Favors Enoxaparin

C Study

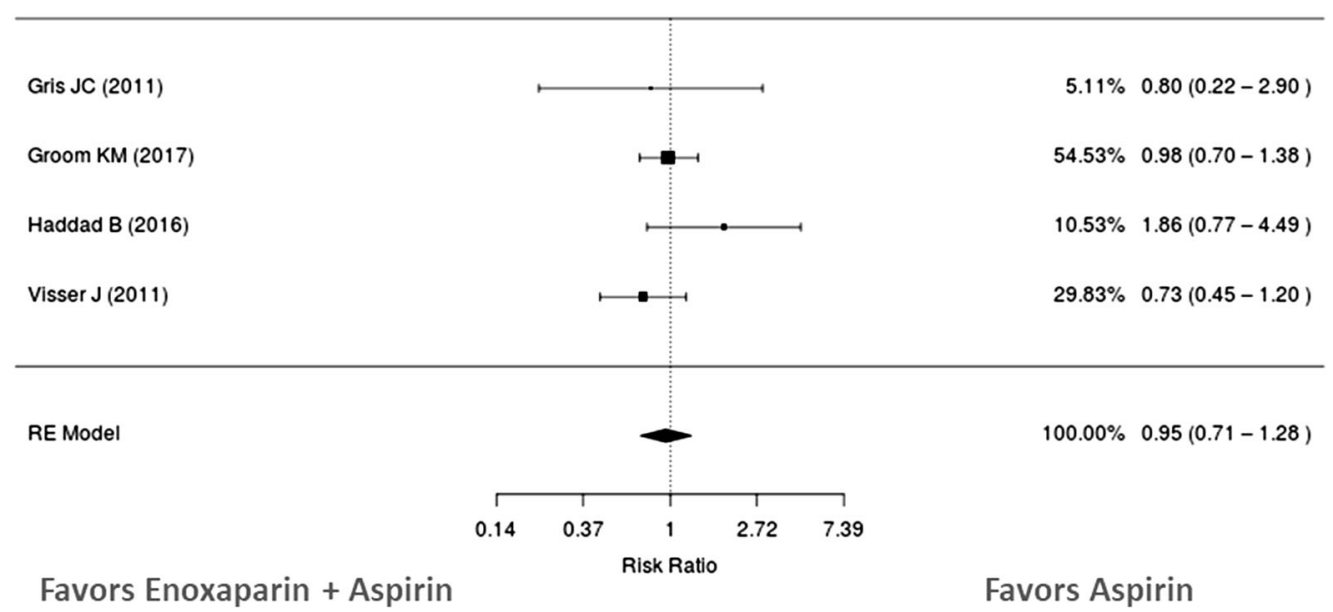

Favors Aspirin

Risk Ratio (95\% CI) 
4Fig. 2 Relative risk of bleeding events with enoxaparin compared to a placebo/no treatment or $\mathbf{b}$ aspirin. $\mathbf{c}$ Relative risk of bleeding events with enoxaparin + aspirin compared to aspirin

In terms of safety, the incidence of thromboembolic events was rare, with only two antenatal cases of DVT with enoxaparin reported in a single study. More bleeding events occurred with enoxaparin than placebo/untreated controls, but the risk of bleeding was lower compared to aspirin. Risk of pregnancy loss was lower with enoxaparin compared to placebo/untreated controls, aspirin, and UFH plus aspirin. Statistically significant reduction in risk of pregnancy loss was shown for enoxaparin plus aspirin compared to aspirin alone. Few studies reported thrombocytopenia and congenital malformations occurring with enoxaparin, the majority reporting only one or two events.

In terms of safety, anticoagulant treatment with enoxaparin is expected to have higher bleeding rates when compared to placebo. A recent systematic review and meta-analysis conducted by Sirico et al. [46] included data from 22,162 pregnant women, of whom 1320 (6\%) were administered LMWH. Although LMWH-treated patients had a higher risk of postpartum hemorrhage, no difference in mean blood loss or risk of blood transfusion at delivery was reported. The authors attributed the higher risk for postpartum hemorrhage to LMWH usage in spite of there being no difference in mean blood loss or in risk of blood transfusion at delivery but noted "the small number of women included in the secondary analysis (784 for mean blood loss at delivery, 883 for blood transfusion) compared to the women included in the primary analysis." Moreover, the inclusion of retrospective cohort data and the lack of adjustments for confounders do impact the generalizability and conclusions of this study. The increased bleeding in enoxaparin-treated patients compared to placebo observed in our review, together with the findings in the review by Sirico et al. [46], supports the practice of assessing risk/benefit when prescribing enoxaparin in pregnancy; caution is required when using enoxaparin in pregnancy and is a trade-off between maternal bleeding and prevention of VTE.

In terms of safety, enoxaparin was associated with a reduction in pregnancy loss in this analysis, which is an unexpected result, as a significant benefit of LMWH for prevention of recurrent pregnancy loss has not been observed in other systematic reviews and meta-analyses $[47,48]$. Observational data and RCTs were included in our review, and this finding must be interpreted cautiously as a safety signal rather than an efficacy signal. Adequately powered robust clinical studies are required to demonstrate the efficacy of enoxaparin to prevent recurrent pregnancy loss in patients with acquired or inherited thrombophilia. One such ongoing study is ALIFE-2, a robust RCT, which is investigating the efficacy of enoxaparin in preventing pregnancy loss in women with inherited thrombophilia and recurrent miscarriage [49]. There is evidence that enoxaparin does not benefit patients with pregnancy loss unrelated to thrombophilia and the practice of treating women with enoxaparin for pregnancy loss unrelated to thrombophilia may be futile, and pose an unnecessary risk to the mother [38].

Fetal safety is of particular concern for all drugs administered during pregnancy. Considering that many women who receive enoxaparin treatment during pregnancy are already at high risk for poor pregnancy outcome, we did not find an incremental fetal risk that was attributable to enoxaparin. The risk of congenital malformations was low and similar to those reported by Shlomo et al. [50] in a populationbased, retrospective cohort study of maternal and infant hospital records between 1998 and 2009. Of the 109,473 singleton pregnancies reported on, 418 and 572 were exposed to enoxaparin during the first and third trimesters, respectively. Importantly, exposure to enoxaparin during the first trimester of pregnancy was not associated with an increased risk of major congenital malformations [adjusted odds ratio (aOR) 1.1, 95\% CI 0.8-1.6], while exposure during the third trimester was not associated with an increased risk of low birth weight, low 
Risk Ratio (95\% CI)

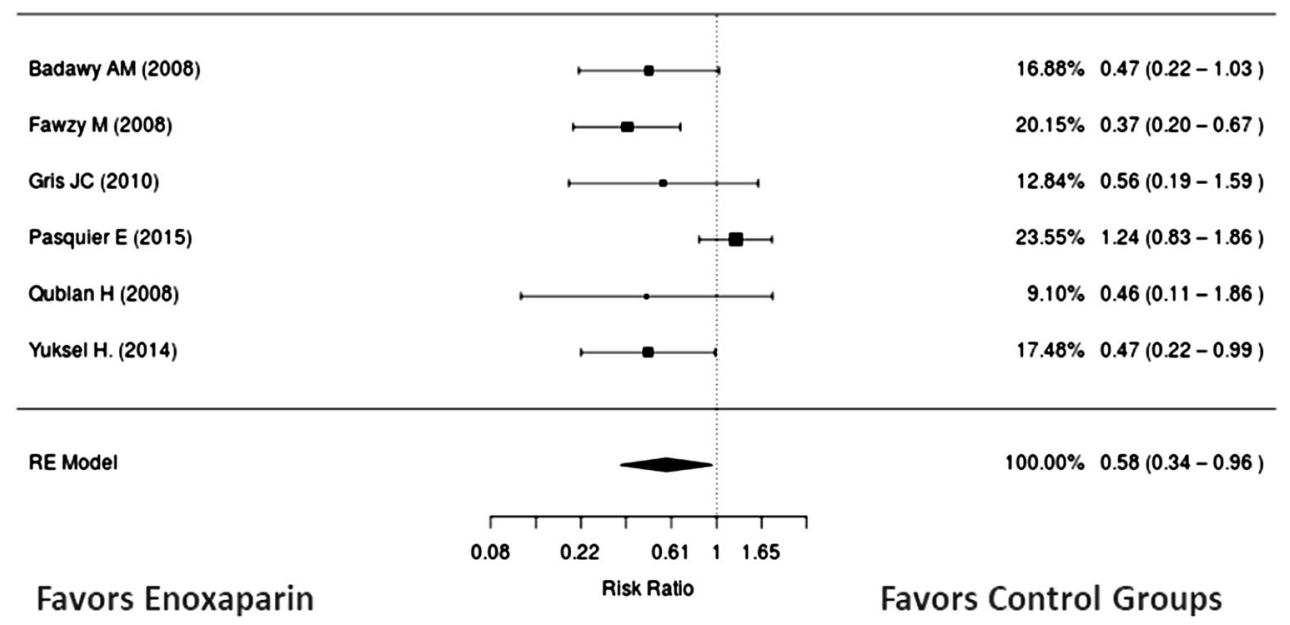

b Study

Risk Ratio (95\% CI)

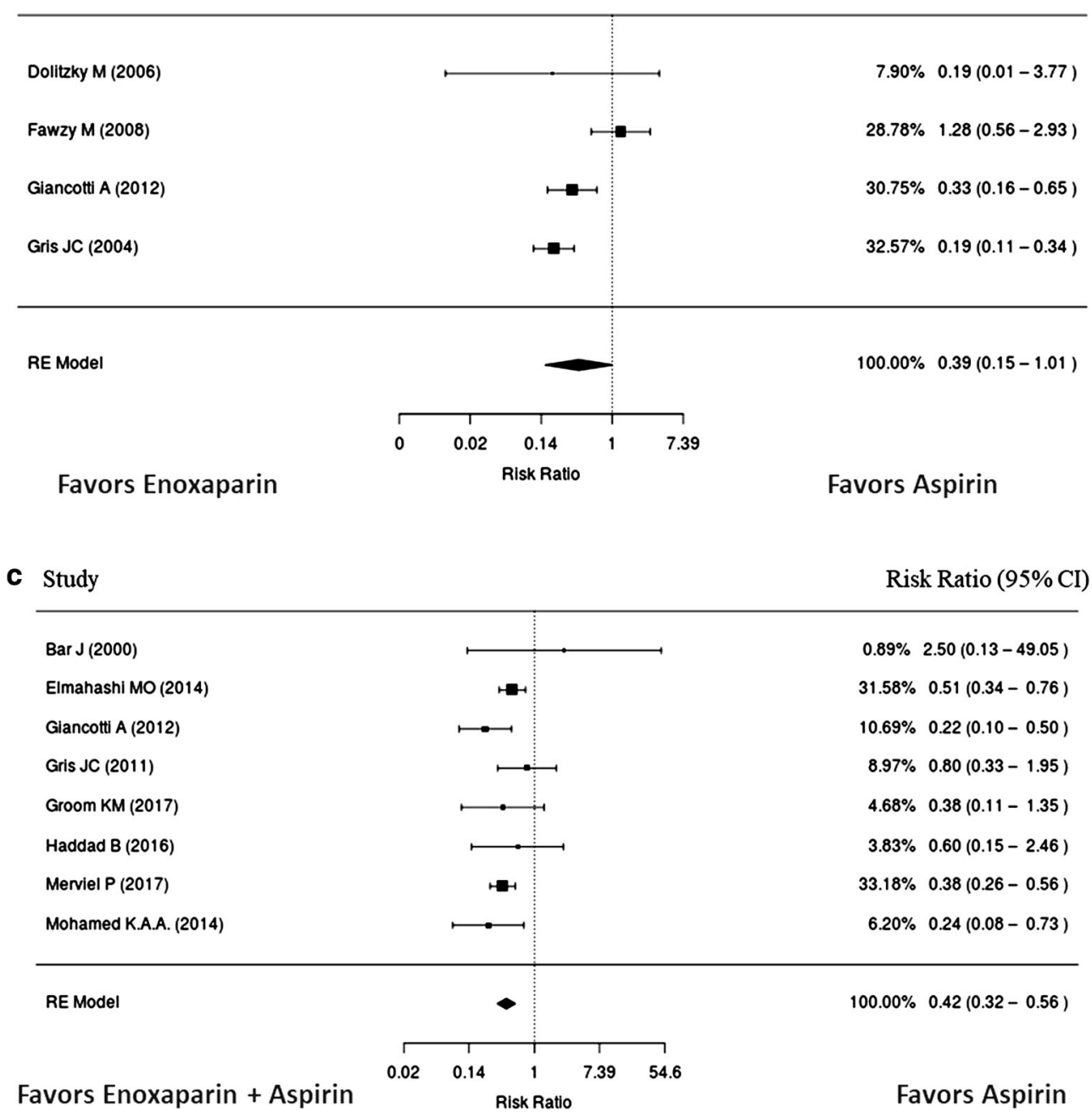


4Fig. 3 Relative risk of pregnancy loss with enoxaparin compared to a placebo/no treatment or $\mathbf{b}$ aspirin. $\mathbf{c}$ Relative risk of pregnancy loss with enoxaparin + aspirin compared to aspirin

Apgar score, or risk of perinatal mortality. The authors concluded that "exposure to enoxaparin during pregnancy was not associated with an increased risk of major malformations in general or according to organ systems. Nonetheless, the risk for specific malformations cannot be ruled out."

In our opinion, the low number of reported congenital malformations with enoxaparin in the literature has biological plausibility, given that enoxaparin has not been shown to cross the placenta in clinical studies that have directly evaluated whether enoxaparin crosses the placenta in vivo [51, 52]. Forestier et al. [51] studied anti-Xa and anti-IIa activities in maternal and fetal blood after enoxaparin administration in five cases of medical abortion during the second trimester (week 23 of gestation); $3 \mathrm{~h}$ after the subcutaneous injection of 7500 anti$\mathrm{Xa}$ IU of enoxaparin, anti-Xa and anti-IIa activities in the five fetuses were not modified compared to controls. Dimitrakakis et al. [52] studied the fetal and maternal effects of enoxaparin $40 \mathrm{mg}$ (4000 IU) in 14 pregnant women and 10 controls due for termination of their pregnancies because of the presence of a major congenital malformation; in the 14 patients receiving drug, no anti-IIa or anti-Xa activities were observed $3 \mathrm{~h}$ post-dose in ultrasound-guided fetal blood samples.

Ideally, the safety and efficacy of enoxaparin in pregnancy should be determined in an adequately powered, randomized, double-blind controlled trial. This study would, however, require an exceptionally large number of patients given the low number of reported events. It may also be challenging to conduct a study because of the large sample size, and ethical justification for such a trial given that enoxaparin has become standard of care internationally and one may argue that clinical equipoise does not exist. A more pragmatic approach would be to supplement the current body of scientific evidence with data from registry studies and pharmacovigilance reports.
Only published studies were included, and therefore the review may be limited by publication bias. The long-term effect of enoxaparin exposure during pregnancy on neurodevelopment requires substantial follow-up and was not reported in the included studies. The gray literature was not searched, and very rare adverse events may not have been even reported. Studies of pregnant patients with mechanical heart valves were excluded because these patients may have altered enoxaparin pharmacokinetics and pharmacodynamics, when compared to pregnant patients without mechanical heart valves and may benefit from a separate systematic review. Therefore, the findings of this systematic review and meta-analysis are not generalizable to pregnant patients with mechanical valves. Also, given the rarity of outcome events, we included both RCT and observational data, which may present certain limitations for analysis.

\section{CONCLUSION}

In terms of safety, enoxaparin use in pregnancy was associated with a significantly lower occurrence of pregnancy loss when compared with control groups and similar rates of bleeding when compared with aspirin; reports of thromboembolic events, thrombocytopenia, and teratogenicity were rare. The efficacy of LMWH for the treatment of VTE is already established, but evidence for enoxaparin specifically remains limited. In light of the adoption of antenatal LMWH into clinical practice, real-world data and continued pharmacovigilance are needed to further inform treatment guidelines on the clinical benefits and risks associated with this form of therapy.

\section{ACKNOWLEDGEMENTS}

Funding. The systematic search and analysis assistance was provided by Doctor Evidence and funded by Sanofi, including open access and publishing fees. 
Medical Writing Assistance. Medical writing support was provided by Jung Min Han and Saranya Chandrudu of Doctor Evidence with publication support by Angelica Stamegna and funded by Sanofi.

Authorship. All named authors meet the International Committee of Medical Journal Editors (ICMJE) criteria for authorship for this article, take responsibility for the integrity of the work as a whole, and have given their approval for this version to be published.

Authorship Contributions. Design of Study and Conceptualization: PN, JS, DP, TS, BJ, RL. Data Curation and Analysis: DP, TS. Original Draft Construction: PN, DP, TS, RL. Draft Review and Scientific Revisions: BJ, VR, RL, JS. Final draft approval: BJ, VR, DP, TS, JS, PN, RL.

Disclosures. Barry Jacobson reports having received speaker fees/honoraria from Sanofi, Bayer, Shire, and Aspen, outside of the contents of this work. Virendra Rambiritch reports previous speaking engagements with Sanofi, outside of the contents of this work. Tobias Sayre was an employee of Doctor Evidence at the time of this analysis. Dara Paek was an employee of Doctor Evidence at the time of this analysis. Poobalan Naidoo is an employee of Sanofi. Jenny Shan is an employee of Sanofi. Rory Leisegang has nothing to disclose.

Compliance with Ethics Guidelines. This review is based on previously conducted studies and does not contain any studies with human participants or animals performed by any of the authors.

Data Availability. Full datasets generated during and/or analyzed during the current study are available from the corresponding author on reasonable request. Additional data are available in the supplementary materials.

Open Access. This article is distributed under the terms of the Creative Commons Attribution-NonCommercial 4.0 International License (http://creativecommons.org/licenses/ by-nc/4.0/), which permits any noncommercial use, distribution, and reproduction in any medium, provided you give appropriate credit to the original author(s) and the source, provide a link to the Creative Commons license, and indicate if changes were made.

\section{REFERENCES}

1. Heit JA, Kobbervig CE, James AH, Petterson TM, Bailey KR, Melton LJ. Trends in the incidence of venous thromboembolism during pregnancy or postpartum: a 30-year population-based study. Ann Intern Med. 2005;143(10):697-706.

2. Pomp E, Lenselink A, Rosendaal F, Doggen CJM. Pregnancy, the postpartum period and prothrombotic defects: risk of venous thrombosis in the MEGA study. J Thromb Haemost. 2008;6(4):632-7.

3. Knight M BK, Tuffnell D, Jayakody H, editors on behalf of MBRRACE-UK. Saving Lives, Improving Mothers' Care: Lessons learned to inform maternity care from the UK and Ireland Confidential Enquiries into Maternal Deaths and Morbidity 2014-16. Oxford: National Perinatal Epidemiology Unit, University of Oxford; 2018.

4. Petersen EE, Davis NL, Goodman D, et al. Vital signs: pregnancy-related deaths, United States, 2011-2015, and strategies for prevention, 13 States, 2013-2017. Morb Mortal Wkly Rep. 2019;68(18):423.

5. Hirsh J, Warkentin TE, Raschke R, Granger C, Ohman EM, Dalen JE. Heparin and low-molecularweight heparin: mechanisms of action, pharmacokinetics, dosing considerations, monitoring, efficacy, and safety. Chest. 1998;114(5):489S-510S.

6. Ostrea EM, Mantaring JB, Silvestre MA. Drugs that affect the fetus and newborn infant via the placenta or breast milk. Pediatr Clin. 2004;51(3):539-79.

7. American College of Obstetricians and Gynecologists. ACOG Practice Bulletin No. 196: thromboembolism in Pregnancy. Obstet Gynecol. 2018;132(1):e1-17. https://doi.org/10.1097/AOG. 0000000000002706 .

8. Bates SM, Greer IA, Middeldorp S, Veenstra DL, Prabulos A-M, Vandvik PO. VTE, thrombophilia, antithrombotic therapy, and pregnancy: antithrombotic therapy and prevention of thrombosis: American College of Chest Physicians evidence-based clinical practice guidelines. Chest. 2012;141(2):e691S-736S. 
9. Bates SM, Rajasekhar A, Middeldorp S, et al. American Society of Hematology 2018 guidelines for management of venous thromboembolism: venous thromboembolism in the context of pregnancy. Blood Adv. 2018;2(22):3317-59. https://doi.org/10. 1182/bloodadvances.2018024802.

10. Chan WS, Rey E, Kent NE, et al. Venous thromboembolism and antithrombotic therapy in pregnancy. J Obstet Gynaecol Can. 2014;36(6):527-53.

11. Royal College of Obstetricians and Gynaecologists. Reducing the risk of thrombosis and embolism during pregnancy and the puerperium. Green-top Guideline. 2015(37a). London: College of Obstetricians and Gynaecologists.

12. Royal College of Obstetricians and Gynaecologists. Thromboembolic disease in pregnancy and the puerperium: acute management. Green-top Guideline. 2015(37b). London: College of Obstetricians and Gynaecologists.

13. Regitz-Zagrosek V, Roos-Hesselink JW, Bauersachs J, et al. 2018 ESC Guidelines for the management of cardiovascular diseases during pregnancy. Eur Heart J. 2018;39(34):3165-241. https://doi.org/10.1093/ eurheartj/ehy340.

14. Greer IA, Nelson-Piercy C. Low-molecular-weight heparins for thromboprophylaxis and treatment of venous thromboembolism in pregnancy: a systematic review of safety and efficacy. Blood. $2005 ; 106(2): 401-7$.

15. Centre for Reviews and Dissemination. CRD's guidance for undertaking reviews in health care. Layerthorpe: University of York, Centre for Reviews and Dissemination; 2009.

16. NICE. Guide to the methods of technology appraisal. London: National Institute for Health and Clinical Excellence (NICE); 2008.

17. Higgins J, Green S, editors. Cochrane handbook for systematic reviews of interventions version 5.1. 0 . The Cochrane Collaboration. 2011. 2015.

18. Moher D, Liberati A, Tetzlaff J, Altman DG. Preferred reporting items for systematic reviews and meta-analyses: the PRISMA statement. Ann Intern Med. 2009;151(4):264-9.

19. Mazzone PJ, Silvestri GA, Patel S, et al. Screening for lung cancer: chest guideline and expert panel report. Chest. 2018;153(4):954-85.

20. Higgins JP, Altman DG, Gøtzsche PC, et al. The Cochrane Collaboration's tool for assessing risk of bias in randomised trials. BMJ. 2011;343:d5928.
21. Wells G. The Newcastle-Ottawa Scale (NOS) for assessing the quality of nonrandomized studies in meta-analysis. http://www.ohrica/programs/clinical_ epidemiology/oxfordhtm. 2013. Accessed 30 Aug 2018.

22. Badawy A, Khiary M, Sherif L, Hassan M, Ragab A, Abdelall I. Low-molecular weight heparin in patients with recurrent early miscarriages of unknown aetiology. J Obstet Gynaecol. 2008;28(3):280-4.

23. Clark P, Walker ID, Langhorne P, et al. SPIN: the Scottish Pregnancy Intervention Study: a multicentre randomised controlled trial of low molecular weight heparin and low dose aspirin in women with recurrent miscarriage. Blood. 2010;115(21):4162-7.

24. Dolitzky M, Inbal A, Segal Y, Weiss A, Brenner B, Carp H. A randomized study of thromboprophylaxis in women with unexplained consecutive recurrent miscarriages. Fertil Steril. $2006 ; 86(2): 362-6$.

25. Elmahashi MO, Elbareg AM, Essadi FM, Ashur BM, Adam I. Low dose aspirin and low-molecularweight heparin in the treatment of pregnant Libyan women with recurrent miscarriage. BMC Res Notes. 2014;7(1):23.

26. Fawzy M, Shokeir T, El-Tatongy M, Warda O, ElRefaiey A-AA, Mosbah A. Treatment options and pregnancy outcome in women with idiopathic recurrent miscarriage: a randomized placebo-controlled study. Arch Gynecol Obstet. 2008;278(1):33-8.

27. Fouda UM, Sayed AM, Abdou AMA, Ramadan DI, Fouda IM, Zaki MM. Enoxaparin versus unfractionated heparin in the management of recurrent abortion secondary to antiphospholipid syndrome. Int J Gynecol Obstet. 2011;112(3):211-5.

28. Gates S, Brocklehurst P, Ayers S, Bowler U. Thromboprophylaxis and pregnancy: two randomized controlled pilot trials that used low-molecularweight heparin. Am J Obstet Gynecol. 2004;191(4):1296-303.

29. Giancotti A, Torre RL, Spagnuolo A, et al. Efficacy of three different antithrombotic regimens on pregnancy outcome in pregnant women affected by recurrent pregnancy loss. J Maternal Fetal Neonatal Med. 2012;25(7):1191-4.

30. Gris J-C, Chauleur C, Faillie J-L, et al. Enoxaparin for the secondary prevention of placental vascular complications in women with abruptio placentae. Thromb Haemost. 2010;103(04):771-9. 
31. Gris J-C, Chauleur C, Molinari N, et al. Addition of enoxaparin to aspirin for the secondary prevention of placental vascular complications in women with severe pre-eclampsia. Thromb Haemost. 2011;105(06):1053-61.

32. Gris J-C, Mercier E, Quéré I, et al. Low-molecularweight heparin versus low-dose aspirin in women with one fetal loss and a constitutional thrombophilic disorder. Blood. 2004;103(10):3695-9.

33. Groom KM, McCowan LM, Mackay LK, et al. Enoxaparin for the prevention of preeclampsia and intrauterine growth restriction in women with a history: a randomized trial. Am J Obstet Gynecol. 2017;216(3):296.e1-14.

34. Haddad B, Winer N, Chitrit Y, et al. Enoxaparin and aspirin compared with aspirin alone to prevent placenta-mediated pregnancy complications. Obstet Gynecol. 2016;128(5):1053-63.

35. Khan E, Basharat A, Jamil M, Ayub S, Khan M. Preventive role of low-molecular-weight heparin in unexplained recurrent pregnancy loss. S Afr J Obstet Gynaecol. 2017;23(1):17-9.

36. Mohamed KAA, Saad AS. Enoxaparin and aspirin therapy for recurrent pregnancy loss due to antiphospholipid syndrome (APS). Middle East Fertil Soc J. 2014;19(3):176-82.

37. Noble LS, Kutteh WH, Lashey N, Franklin RD, Herrada J. Antiphospholipid antibodies associated with recurrent pregnancy loss: prospective, multicenter, controlled pilot study comparing treatment with low-molecular-weight heparin versus unfractionated heparin. Fertil Steril. 2005;83(3):684-90.

38. Pasquier E, de Saint Martin L, Bohec C, et al. Enoxaparin for prevention of unexplained recurrent miscarriage: a multicenter randomized doubleblind placebo-controlled trial. Blood. 2015;125(14):2200-5.

39. Qublan H, Amarin Z, Dabbas M, et al. Low-molecular-weight heparin in the treatment of recurrent IVF-ET failure and thrombophilia: a prospective randomized placebo-controlled trial. Human Fertil. 2008;11(4):246-53.

40. Urman B, Ata B, Yakin K, et al. Luteal phase empirical low molecular weight heparin administration in patients with failed ICSI embryo transfer cycles: a randomized open-labeled pilot trial. Hum Reprod. 2009;24(7):1640-7.

41. Visser J, Ulander V-M, Helmerhorst FM, et al. Thromboprophylaxis for recurrent miscarriage in women with or without thrombophilia. Thromb Haemost. 2011;105(02):295-301.
42. Bar J, Cohen-Sacher B, Hod M, Blickstein D, Lahav J, Merlob P. Low-molecular-weight heparin for thrombophilia in pregnant women. Int J Gynecol Obstet. 2000;69(3):209-13.

43. Sergio F, Maria Clara DA, et al. Prophylaxis of recurrent preeclampsia: low-molecular-weight heparin plus low-dose aspirin versus low-dose aspirin alone. Hypertens Pregnancy. 2006;25(2):115-27.

44. Merviel P, Cabry R, Lourdel E, et al. Comparison of two preventive treatments for patients with recurrent miscarriages carrying a C677T methylenetetrahydrofolate reductase mutation: 5-year experience. J Int Med Res. 2017;45(6):1720-30.

45. Yuksel H, Kayatas S, Boza AT, Api M, Ertekin AA, Cam C. Low molecular weight heparin use in unexplained recurrent miscarriage. Pak J Med Sci. 2014;30(6):1232.

46. Sirico A, Saccone G, Maruotti GM, et al. Low molecular weight heparin use during pregnancy and risk of postpartum hemorrhage: a systematic review and meta-analysis. J Maternal Fetal Neonatal Med. 2019;32(11):1893-1900.

47. de Jong PG, Kaandorp S, Di Nisio M, Goddijn M, Middeldorp S. Aspirin and/or heparin for women with unexplained recurrent miscarriage with or without inherited thrombophilia. Cochrane Database Syst Rev. 2014. https://doi.org/10.1002/ 14651858.cd004734.pub4.

48. Zhang T, Ye X, Zhu T, et al. Antithrombotic treatment for recurrent miscarriage: Bayesian network meta-analysis and systematic review. Medicine (Baltimore). 2015;94(45):e1732. https://doi.org/10. 1097/MD.0000000000001732.

49. de Jong PG, Quenby S, Bloemenkamp KW, et al. ALIFE2 study: low-molecular-weight heparin for women with recurrent miscarriage and inherited thrombophilia-study protocol for a randomized controlled trial. Trials. 2015;16(1):208.

50. Shlomo M, Gorodischer R, Daniel S, et al. The fetal safety of enoxaparin use during pregnancy: a population-based retrospective cohort study. Drug Saf. 2017;40(11):1147-55.

51. Forestier F, Daffos F, Capella-Pavlovsky M. Low molecular weight heparin (PK 10169) does not cross the placenta during the second trimester of pregnancy study by direct fetal blood sampling under ultrasound. Thromb Res. 1984;34(6):557-60.

52. Dimitrakakis C, Papageorgiou P, Papageorgiou I, Antzaklis A, Sakarelou N, Michalas S. Absence of transplacental passage of the low molecular weight heparin enoxaparin. Pathophysiol Haemost Thromb. 2000;30(5):243-8. 